\title{
Regulating cell volume
}

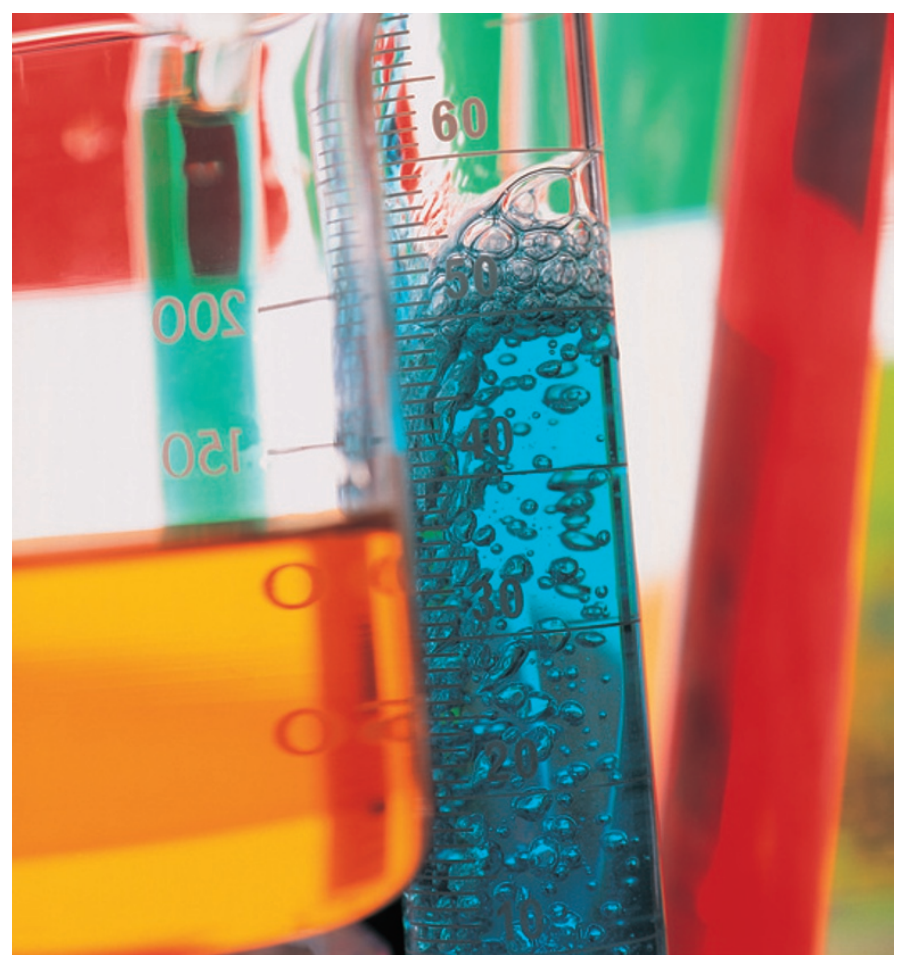

DigitalVision/PunchStock

Two groups
now identify
Leu-rich
repeat-
containing
protein $8 \mathrm{~A}$
(LRRC8A) as
an essential
component of
the VRAC

Lexpressing a fluorescent protein, the fluorescence of which decreases

Cells must maintain a constant volume, despite the rapid influx of water that will cause them to swell in response to changes in osmolarity. The volume-regulated anion channel (VRAC) promotes volume decrease in response to cell swelling by conducting the efflux of ions, other osmolytes and, consequently, water, but its molecular identity has been elusive. Two groups now identify Leu-rich repeat-containing protein 8A (LRRC8A) as an essential component of the VRAC.

To identify genes that encode the VRAC or its regulators, Qiu et al. and Voss et al. carried out genomewide RNAi screens in human cells

when cell swelling causes the VRAC channels to open. Focusing on proteins that are predicted to harbour a transmembrane domain they found that knockdown of $L R R C 8 A$ sustained fluorescence on cell swelling, which suggests that LRRC8A is part of the VRAC or is required for its activation. Indeed, both groups also showed that knockdown of $L R R C 8 A$ suppressed $\mathrm{I}_{\mathrm{Cl}(\text { swell) }}$ activity (a current of $\mathrm{Cl}^{-}$ions that the VRAC predominantly activates in response to swelling) in patch-clamp assays. Qiu et al. renamed this poorly characterized gene SWELL1.

Qiu et al. and Voss et al. observed that LRRC8A is located in the plasma membrane and that its Leu-rich repeats are cytoplasmic. They also assessed whether the four homologues of LRRC8A (LRRC8BLRRC8E) regulate the VRAC. Qiu et al. found that knockdown of each homologue had little effect in their RNAi screen, which suggests that they are not essential for VRAC activity on their own. In agreement, Voss et al. found that, with the exception of $L R R C 8 A$, the disruption of single LRRC8 genes using clustered, regularly interspaced short palindromic repeats (CRISPR)-Cas did not abolish $\mathrm{I}_{\mathrm{Cl}(\text { swell) }}$. However, $\mathrm{I}_{\mathrm{Cl}(\text { swell) }}$ was abolished in cell lines in which all other isoforms (LRRC8B-LRRC8E) were disrupted. Furthermore, in cells in which all five LRRC8 genes were disrupted, $\mathrm{I}_{\mathrm{Cl}(\text { (swell) }}$ was restored by the co-transfection of $L R R C 8 A$ and at least one of the other LRRC8 isoforms; co-immunoprecipitation revealed binding between different LRRC8 isoforms, and the combination of the transfected isoforms determined the kinetics by which $\mathrm{I}_{\mathrm{Cl}(\text { swell) }}$ was inactivated. They concluded that LRRC8 heteromers, which may be hexameric because of their homology to channel-forming pannexins, are an integral part of the VRAC.

Qiu et al. also assessed whether LRRC8A is a component of the VRAC or rather its indirect regulator. The substitution of LRRC8A at residue Thr44 with Cys or Arg altered the selectivity of the VRAC, increasing or decreasing, respectively, its permeability to $\mathrm{I}^{-}$ions. As ion selectively is mostly regulated by the pore of the channel or its associated subunits, this suggests that LRRC8A is part of the VRAC pore.

Finally, both groups noticed that swelling-induced efflux of the osmolyte taurine was abolished in cells in which LRRC8A was disrupted. Voss et al. showed that this could be rescued by co-transfection of $L R R C 8 A$ and $L R R C 8 C$. That efflux of the osmolyte taurine was dependent on LRRC8 heteromers indicates that the VRAC is identical to the volume-stimulated organic osmolyte and anion channel (VSOAC).

In short, these studies identify LRRC8A as a component of the VRAC; this finding should aid the further molecular characterization of this channel and our understanding of its roles in physiology and disease. Katharine H. Wrighton

ORIGINAL RESEARCH PAPERS Qiu, Z. et al. SWELL1, a plasma membrane protein, is an essential component of volume-regulated anion channel. Cell 157, 447-458 (2014) | Voss, F. K. et al. Identification of LRRC 8 heteromers as an essential component of the volume-regulated anion channel VRAC. Science http://dx.doi.org/10.1126/ science.1252826 (2014) 